\title{
ALLPASS BASED ON A GENERALIZED DIVIDER PRINCIPLE
}

This paper deals with a new allpass structure based on a generalized divider principle. An efficient modification of the basic general divider structure using Antoniou's GICs is presented and the corresponding 1st-and 2nd-order allpass circuits are derived. A detail discussion is devoted to the optimized design of the 2nd-order allpass, taking minimization of the active elements nonidealities and dynamics optimization into account. An original design procedure is described and documented on numerical examples.

\section{Introduction}

All-pass filters represent a meaningful part of modern communication systems, making possible equalization of signal chain group-delay. This is important especially in the case of the datasignal or video-signal processing. As known, the basic properties of the all-pass filters are determined by all-pass transfer function (1)

$$
H(s)=h \frac{v(-s)}{v(s)}=h \frac{a_{0}-a_{1} s+s_{2} s^{2}-\ldots+\left(-1^{n}\right) a_{n} s^{n}}{a_{0}+a_{1} s+a_{2} s^{2}+\ldots+a_{n} s^{n}}
$$

With respect to the stability condition, the polynomial $v(s)$ has to be a Hurwitz polynomial. It is easy to derive that the transfer function (1) corresponds to the non-minimal phase circuit whose magnitude frequency response is frequency independent, determined by a multiplicative factor $h$. Phase response can be expressed in the form (2)

$$
\varphi(\omega)=-2 \arctan \frac{\omega N\left(-\omega^{2}\right)}{M\left(-\omega^{2}\right)},
$$

where $\omega N\left(-\omega^{2}\right) \ldots$ represents an odd part of the polynomial $v(j \omega)$ and

$$
M\left(-\omega^{2}\right) \quad \ldots \text { represents an even part of } v(j \omega) .
$$

The group delay $\tau(\omega)$ is defined by the known formula

$$
\tau(\omega)=-\frac{d \varphi}{d \omega} \text {. }
$$

There are many ways how to realize the transfer function (1). Remember that passive realization of the group-delay equalizer is usually based on the $1^{\text {st }}$ - or $2^{\text {nd }}$-order lattice and their unsymmetrical equivalent circuits - see [1], [2]. In the field of active RC circuits are well-known implementations using a single-amplifier circuit or universal biquad configurations as mentioned in [3], [4], [5] and others. The first group of circuits suffers from inappropriate sensitivity to the element value variations and amplifier non-ide- alities. At the same time, a parameter setting is rather complicated and does not allow simple tuning. The second group shows better sensitivity properties, but negative odd numerator coefficients are obtained as a difference of positive and negative terms. With respect to this, such a circuit is sensitive to the perfect setting and amplifier non-idealities. The mentioned circuits usually show a magnitude frequency response distortion which is caused by coefficient errors, or, in other words, by errors of transfer function pole-zero location.

To find a more suitable solution, a considerable effort has been devoted to the search for a new circuit implementation of the allpass transfer function. As shown in the following section, one of the possible ways is based on a suitable application of a generalized divider principle.

\section{A generalized divider}

In Ref.[6] we have presented a universal filter structure based on a generalized divider principle. The basic arrangement of the mentioned structure is shown in Fig. 1. The "black boxes" marked as GIC correspond to the generalized immitance converters characterized by the $1^{\text {st }}$-order conversion function $k_{i}(s)=k_{i} s$, where $s$ denotes complex frequency. Note that such a structure allows realization of any transfer function by appropriate setting of divider branches, as is evident from the corresponding transfer function (4)

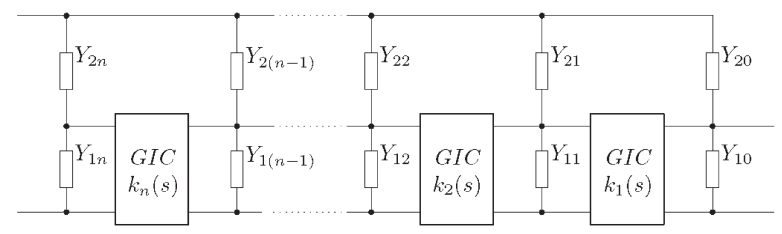

Fig. 1: Generalized divider arrangement

$$
H(s)=\frac{Y_{20}+k_{1}(s)\left[Y_{21}+k_{2}(s)\left[Y_{22}+\ldots+k_{n}(s)\left[Y_{2 n}\right] \ldots\right]\right]}{\left.\left(Y_{10}+Y_{20}\right)+k_{1}(s)\left[\left(Y_{11}+Y_{21}\right)+k_{2}(s)\left[\left(Y_{12}+Y_{22}\right)+\ldots+k_{n}(s)\left[Y_{1 n}+Y_{2 n}\right)\right] \ldots\right]\right]} .
$$

\footnotetext{
* Daša Tichá

Department of Telecommunications, EF, University of Žilina, E-mail: ticha@fel.utc.sk
} 
Comparing $H(s)$ to the general transfer function in the polynomial form (5)

$$
H(s)=\frac{a_{0}+a_{1} s+a_{2} s^{2}+\ldots+a_{n} s^{n}}{d_{0}+d_{1} s+d_{2} s^{2}+\ldots+d_{n} s^{n}},
$$

it is easy to derive formulae for numerator and denominator coefficients as the functions of branch admittances and GICs conversion functions

$$
a_{k}=Y_{2 k} \prod_{i=1}^{k} k_{i} ; d_{k}=\left(Y_{1 k}+Y_{2 k}\right) \prod_{i=1}^{k} k_{i},
$$

where $k_{i}$ - denotes the conversion-function-multiplicative constant of the $i^{\text {th }} \mathrm{GIC}$.

To apply the presented generalized divider structure in the allpass design it is necessary to accept the following restrictions:

- The equal values of numerator and denominator coefficients at the same power of $s$ (but, in general, all the numerator coefficients can be multiplied by the transfer multiplicative constant $\left.h: \Rightarrow a_{i}=h . d_{i}, i=0,1,2, \ldots, n\right)$.

- The odd numerator coefficients have to be negative $\Rightarrow a_{j}=-h \cdot d_{j}, j=1,3,5, \ldots$

The relevant problem can arise in the second condition fulfilment. The original generalized divider structure allows realization of the transfer function containing the positive numerator coefficients only, as is evident from Eq. (4). To obtain the required neg-

$$
Z_{1-1^{\prime}}=\frac{Z_{k 1}}{Z_{k 2}} Z_{x}
$$

Using this principle it is easy to derive the modified generalized divider structure shown in Fig. 3, convenient to the arbitrary allpass transfer realization. The odd "upper" branch admittances $Y_{2 j, j}=1,3,5, \ldots$ are connected to the auxiliary port of the corresponding odd GICs, and, with respect to the GIC transforming effect, they behave as the negative ones. The equivalent admittance of the modified branch is expressed by the equation (9)

$$
Y_{2 j(m)}=-\frac{Z_{2 j}}{Z_{1 j}} Y_{2 j}=\frac{R_{2 j}}{R_{1 j}} Y_{2 j},
$$

where $Y_{2 j(m)}$ - denotes transformed (modified) admittance of $\mathrm{j}^{\text {th }}$ branch,

$Z_{i j}-$ denotes $i^{\text {th }}$ impedance of $j^{\text {th }}$ GIC circuit (see Fig. 2),

$Y_{2 j}$ - denotes the original $\mathrm{j}^{\text {th }}$ branch admittance.

As evident the converter impedance $Z_{k 1}, Z_{k 2}$ should be of the same type to save the character of the transformed admittance $Z_{x}$. It is easy to derive; the correct circuit behaviour requires arrangement of GIC circuit elements as follows:

$$
Z_{k 1}=R_{1} ; Z_{k 2}=R_{2} ; Z_{k 3}=R_{3} ; Z_{k 4}=\frac{1}{s C_{4}} .
$$

The resulting transfer function of the improved circuit structure can be written in the form (11)

$$
H(s)=\frac{Y_{20}-k_{1} \alpha_{1} Y_{21} s+k_{1} k_{2} Y_{22} s^{2}-k_{1} k_{2} k_{3} \alpha_{1} \alpha_{3} Y_{23} s^{3}+\ldots}{\left(Y_{10}+Y_{20}\right)+k_{1}\left(Y_{11}-\alpha_{1} Y_{21}\right) s+k_{1} k_{2}\left(Y_{12}+Y_{22}\right) s^{2}+k_{1} k_{2} k_{3}\left(Y_{13}-\alpha_{1} \alpha_{3} Y_{23}\right) s^{3} \ldots},
$$

ative odd coefficients, it is necessary to change the sign of the corresponding branch admittances, or, to use inverted source voltage at the inputs of the upper odd branches. An efficient solution offers the use of the known Antoniou's GIC circuitry - see [4, 5]. Let us consider Antoniou's GIC in Fig. 2, whose conversion function is expressed by Eq. (7), and now modify the original circuit by adding the auxiliary port $x-x^{\prime}$. An arbitrary loading impedance $Z_{x}$, connected to the auxiliary port is transformed to the "main" input port $1-1^{\prime}$ as the negative impedance $Z_{1-1}$, which is presented by the formula (8)

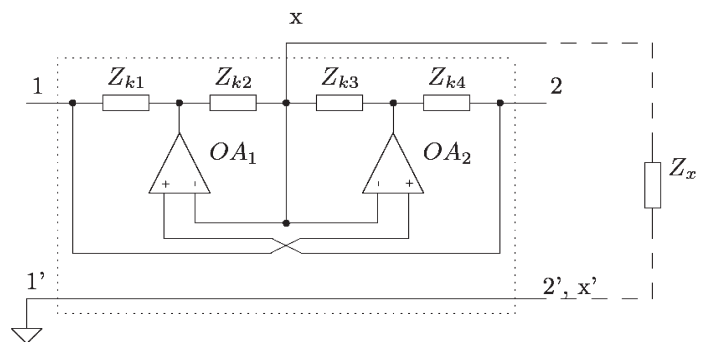

Figure 2: Antoniou's GIC circuit

$$
k=\frac{Z_{k 1} Z_{k 3}}{Z_{k 2} Z_{k 4}}
$$

where $\alpha_{\mathrm{j}}$ - expresses the ratio $\alpha_{j}=R_{2 j} / R_{1 j}, j=1,3,5, \ldots$ of $i^{\text {th }}$ GIC impedances.

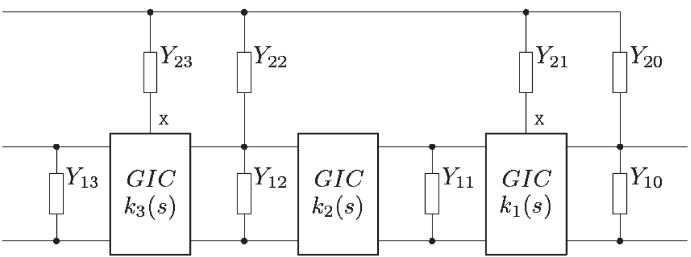

Fig. 3: Modified divider structure

As evident, the odd transfer function coefficients are now expressed in the form (12), while the even coefficients remain unchanged and correspond to the expression (6).

$$
\begin{aligned}
& a_{j}=Y_{2 j} \prod_{l=1,3,5 . .}^{j} \alpha_{l} \prod_{k=1}^{j} k_{k} ; d_{j}=\left(Y_{1 j}-Y_{2 j}\right) \prod_{l=1,3,5 . .}^{j} \alpha_{l} \prod_{k=1}^{j} k_{k}, \\
& j=1,3,5, \ldots
\end{aligned}
$$

The presented results indicate effective implementation of an arbitrary order allpass transfer function. With respect to the number of circuit elements (divider branches) the structure is canonical. 
Note that the special case for the transfer multiplicative constant $h=1$ leads to the minimum number elements realization. The absolute value coefficient equivalence

$$
\left|a_{i}\right|=\left|d_{i}\right|
$$

compared to the $\operatorname{Eqs}(6,12)$ gives divider branch design conditions

$$
Y_{1 i}=0, i=0,2,4 \ldots \quad Y_{1 j}=2 Y_{2 j}, j=1,3,5 \ldots
$$

It is important to say that the divider principle causes a restriction in transfer multiplicative constant value. The appropriate choice is limited to the unequality $h \leq 1$ and cannot be exceeded by no means.

In the following the particular cases of the $1^{\text {st }}$ - and the $2^{\text {nd }}$ order allpass circuits will be discussed in detail.

\section{The 1 st and 2 nd-order allpass}

The simpliest version of allpass circuit is presented by $1^{\text {st }}$-order divider structure shown in Fig. 4.

The circuit transfer function was found in the form (14)

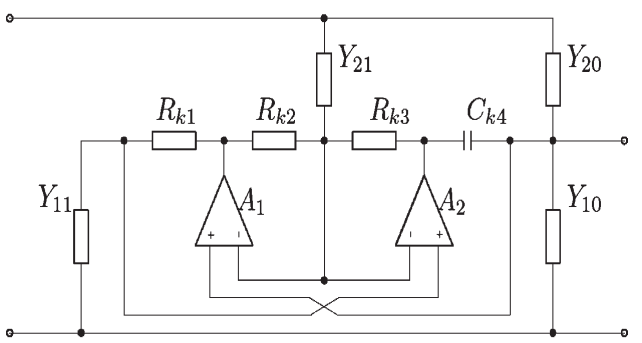

Fig. 4: 1st-order allpass circuit

$$
H(s)=\frac{-Y_{21} k_{1} \alpha_{1} s+Y_{20}}{k_{1}\left(Y_{11}-Y_{21} \alpha_{1}\right) s+Y_{20}+Y_{10}},
$$

where $k_{1}=\frac{R_{k 1} R_{k 3} C_{k 4}}{R_{k 2}}$.

A comparison of the expression (14) to the general form of $1^{\text {st }}$-order allpass transfer function (15) leads to the simple design equations (16)

$$
\begin{aligned}
& H(s)=h \frac{s-\sigma}{s+\sigma}, \\
& Y_{10}=\sigma k_{1} \alpha_{1} \frac{1-h}{h} Y_{21} ; Y_{11}=\alpha_{1} \frac{h+1}{h} Y_{21} ; \\
& Y_{20}=\sigma k_{1} \alpha_{1} Y_{21} .
\end{aligned}
$$

Here $\alpha_{1}=\frac{R_{2}}{R_{1}} \cdot k_{1}$, and $Y_{21}$ represent free parameters and can be chosen arbitrarily, e.g. with respect to the additional optimized design conditions.
Now, let us devote our attention to the $2^{\text {nd }}$-order allpass. The circuit diagram is shown in Fig. 5, GICs are implemented by the mentioned Antoniou's circuitry - see Fig. 2. Circuit symbolic transfer function is expressed in the "standard" form (17)

$$
H(s)=\frac{a_{2} s^{2}-a_{1} s+a_{0}}{s^{2}+d_{1} s+d_{0}}=h \frac{s^{2}-s \frac{\omega_{0}}{Q}+\omega_{0}^{2}}{s^{2}+s \frac{\omega_{0}}{Q}+\omega_{0}^{2}} .
$$

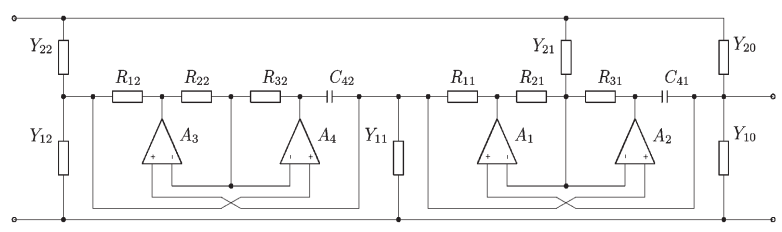

Fig. 5: 2nd-order allpass circuit using modified Antoniou's GIC

The transfer function coefficients are expressed by the formulae

$$
\begin{aligned}
& a_{1}=h \frac{\omega_{0}}{Q}=\frac{\alpha_{1}}{k_{2}} \frac{Y_{21}}{Y_{12}+Y_{22}}, d_{1}=\frac{\omega_{0}}{Q}=\frac{1}{k_{2}} \frac{Y_{11}-\alpha_{1} Y_{21}}{Y_{12}+Y_{22}} \\
& a_{0}=h \omega_{0}^{2}=\frac{1}{k_{1} k_{2}} \frac{Y_{20}}{Y_{12}+Y_{22}}, d_{0}=\omega_{0}^{2}=\frac{1}{k_{1} k_{2}} \frac{Y_{10}+Y_{20}}{Y_{12}+Y_{22}}
\end{aligned}
$$

and

$$
h=\frac{Y_{22}}{Y_{12}+Y_{22}}, k_{i}=\frac{R_{1 i} R_{3 i} C_{4 i}}{R_{2 i}}, \alpha_{i}=\frac{R_{2 i}}{R_{1 i}}, i=1,2
$$

Similarly to the aforementioned $1^{\text {st }}$-order case, it is possible to derive design equations of divider branches $Y_{i k i, k=1,2}$ from Eqs. (18). The result is

$$
\begin{aligned}
& Y_{10}=\frac{1-h}{h} \omega_{0}^{2} k_{1} k_{2} Y_{22}, Y_{11}=\frac{1+h}{h} \frac{\omega_{0}}{Q} k_{2} Y_{22}, \\
& Y_{12}=\frac{1-h}{h} Y_{22}, Y_{20}=\omega_{0}^{2} k_{1} k_{2} Y_{22}, Y_{21}=\frac{\omega_{0}}{Q} \frac{k_{2}}{\alpha_{1}} Y_{22}, \\
& Y_{22} \ldots \text { arbitrary. }
\end{aligned}
$$

Similarly to the $1^{\text {st }}$-order case, the values of $Y_{22}, k_{i}$ and $\alpha_{i}$ parameters are free and can be conformed to the optimum design conditions. In accordance with the previous considerations and derived "basic" design formulae $(16,20)$, the higher-order allpass can be easily created only by adding next branches to the designed circuit.

\section{Optimized design of the $2^{\text {nd }}$-order circuit}

The presented design equations (20) are fully valid in the case of an idealized circuit, i.e. the circuit containing idealized active elements. As known, especially the influence of amplifier finite frequency dependent gain significantly change the circuit behav- 
iour, particularly increase the transfer funcion order and vary "main" poles and zeroes location. From this point-of-view, it is advisable to use the free design parameters to minimize the influence of amplifier non-idealities. A detail analysis of this topic was made in [7], in this place the main results will be summarized and optimized design algorithms presented. $H(s)$

Starting from the allpass general biquadratic transfer function

$$
\begin{aligned}
& H(s)=\frac{N(s)}{D(s)}=h \frac{s^{2}-s \frac{\omega_{0 z}}{Q_{z}}+\omega_{0 z}^{2}}{s^{2}+s \frac{\omega_{0 p}}{Q_{p}}+\omega_{0 p}^{2}}= \\
& =\frac{a_{2} s^{2}-a_{1} s+a_{0}}{s^{2}+d_{1} s+d_{0}} .
\end{aligned}
$$

The design optimization conditions include

- minimization of $\omega_{p}$ and $\omega_{z}$ errors,

- minimization of $Q_{p}$ and $Q_{z}$ errors,

- dynamic optimization, i.e. equalization of maximum output voltages of all the amplifiers.

Note that the optimization conditions strongly depend on the amplifiers type used in Antoniou's converter circuitry, as documented in ref.[8]. With respect to the presented results, the transimpedance amplifier (CFOA) has been chosen as the most suitable for voltage-mode design.

The derivation of general optimization conditions requires to express parameters of the main poles and zeroes in symbolic form. But the transfer function of the real circuit is of the $6^{\text {th }}$-order, considering simple single-pole models of the amplifiers used. A symbolic evaluation of the main poles and zeroes then presents a dificult mathematical task, which can be solved only approximately, despite the modern mathematical software at disposal. The developed general algorithm is based on order reduction of the transfer function numerator and denominator polynomials neglecting the higherorder error terms. It is formed as follows:

1. Symbolic transfer function of the real circuit is computed under consideration of finite, frequency independent amplifier gain.

2. Numerator and denominator of the obtained symbolic transfer function is divided by the highest power of amplifier gain:

$N(s) \Rightarrow \frac{N(s)}{A^{m}}, \quad D(s) \Rightarrow \frac{N(s)}{A^{m}}$,

where $A$ - means amplifier gain, $m$ - denotes the highest power of $A$.

3. All the terms of recalculated numerator and denominator poly nomials containing power of A higher than 1 are neglected, i.e.

$$
\frac{a_{i}}{A^{k}}, k>1 \rightarrow 0 \text { and } \frac{d_{j}}{A^{k}}, k>1 \rightarrow 0 .
$$

Then

$$
N^{\prime}(s)=s^{2}+a_{11} s+a_{01}+\frac{1}{A}\left(a_{12} s+a_{02}\right),
$$

$D^{\prime}(s)=s^{2}+d_{11} s+s_{01}+\frac{1}{A}\left(d_{12} s+d_{02}\right)$.

4. Gain amplifier symbol $A$ in $N^{\prime}(s)$ and $D^{\prime}(s)$ is replaced by a frequency dependent relationship

$A \Rightarrow A_{0} \frac{\omega_{x}}{s+\omega_{x}} \doteq \frac{B}{s}$

where $B=A_{0} \omega_{x}$, and $\omega_{x}$ means dominant pole of amplifier gain frequency response.

5. Expressions of $N_{0}(s)$ and $D_{0}(s)$ are formally rearranged into a polynomial form and used for simplified transfer function $H_{0}(s)$ compilation

$$
H^{\prime}(s)=h^{\prime} \frac{N^{\prime}(s)}{D^{\prime}(s)}=h^{\prime} \frac{s^{3}+a_{2}^{\prime} s^{2}+a_{1}^{\prime} s+a_{0}^{\prime}}{s^{3}+d_{2}^{\prime} s^{2}+d_{1}^{\prime} s+d_{0}^{\prime}}
$$

6. Numerator and denominator polynomials $N^{\prime}(s)$ and $D^{\prime}(s)$ are decomposed into

$$
\begin{aligned}
& N^{\prime}(s)=s^{3}+a_{2}^{\prime} s^{2}+a_{1}^{\prime} s+a_{0}^{\prime}= \\
& =\left(s+\sigma_{z}\right)\left(s^{2}+a_{e 1} s+a_{e 0}\right)= \\
& =\left(s+\sigma_{z}\right)\left(s^{2}+s \frac{\omega_{0 z}^{\prime}}{Q_{z}^{\prime}}+\omega_{0 z}^{\prime 2}\right) . \\
& D^{\prime}(s)=s^{3}+d_{2}^{\prime} s^{2}+d_{1}^{\prime} s+d_{0}^{\prime}= \\
& =\left(s+\sigma_{p}\right)\left(s^{2}+d_{e 1} s+d_{e 0}\right)= \\
& =\left(s+\sigma_{p}\right)\left(s^{2}+s \frac{\omega_{0 p}^{\prime}}{Q_{p}^{\prime}}+\omega_{0 p}^{\prime 2}\right) .
\end{aligned}
$$

Here $\omega_{0 p}^{\prime}, Q_{p}^{\prime}$ represent parameters of "real" main poles, $\omega_{0 z}^{\prime}$, $Q_{z}^{\prime}$ "real" main zeroes. $\sigma_{p}$ and $\sigma_{z}$ represent auxiliary "equivalent" real pole and zero without any importance for the following optimization procedure.

The third-order polynomial decomposition is possible using mathematical programs, or, in the simplified way using approximate formulae

$$
\begin{aligned}
& \sigma_{p}=d_{2}^{\prime}-\frac{\omega_{0 p i d}}{Q_{p i d}} ; \omega_{0 p}^{\prime 2}=d_{e 0}=\frac{d_{0}^{\prime}}{\sigma_{p}} \\
& d_{e 1 A}=\frac{d_{1}^{\prime}-\omega_{0 p i d}^{2}}{\sigma_{p}}, \text { or } d_{1 e B}=\frac{d_{2}^{\prime} d_{1}^{\prime}-d_{1}^{\prime} d_{1 i d}-d_{0}^{\prime}}{\sigma_{p}^{2}},
\end{aligned}
$$

where $d_{1 i d}=\frac{\omega_{0 p i d}}{Q_{p i d}}, \omega_{0 p i d}, Q_{p i d}$ are parameters of the idealized transfer function (17), resp. (21), expressed in the symbolic form. The parameter $Q_{p}^{\prime}$ can be evaluated from Eq. (26)

$$
Q_{p}^{\prime}=\frac{\omega_{p}^{\prime}}{d_{e 1}} .
$$


Note that the presented simplified equations are valid under assumption

$$
\sigma_{p}>>\omega_{0 p}, \omega_{0 p} \doteq \omega_{0 p i d}
$$

for case $A$, or $d_{e 1} \doteq d_{1 i d}$ for case $B$. Similar equations and assumptions can be used for the evaluation of the equivalent numerator parameters $\sigma_{z}, \omega_{0 z}^{\prime}$ and $Q^{\prime}$. As shown in Ref. [7], the sufficient accuracy is achieved when $B / \omega_{0}>10^{1}$, with respect to the $Q$ factor. The solved numerical examples confirmed small evaluation errors of $d_{e 0}=\omega_{02}^{\prime}$ parameters, even in the case of lower ratios $B / \omega_{0}$. In the case of parameter $d_{e 1}$ or $a_{e 1}$ evaluation accuracy, case $A$ results show higher values, case $B$ results give lower values of computed $Q$ - factor in comparison to the exact values. The acceptable approximation is the average value, i.e.

$$
d_{e 1 A B}=\frac{1}{2}\left(d_{e 1 A}+d_{e 1 B}\right)
$$

where the resulting error of the computed $Q$ parameter is under $1 \%$ for ratio $B / \omega_{0} \leq 15$.

The necessary circuit symbolic analysis and all the symbolic evaluations including the derivation of symbolic simplified transfer function parameters were made using mathematical program MAPLE V, release 5.

The optimization procedure alone is based on utilization of circuit degrees of freedom given by an additional number of optional circuit elements in comparison to the number of given design parameters. In the considered case, the optimized circuit has eight degrees of freedom, with respect to the six given parameters of the transfer function (21) and 14 optional passive elements. In the following, the procedure will be discussed in detail and the results demonstrated on typical examples.

The basic stage of the optimization procedure includes minimization of the simplified transfer function coefficient errors $(27)^{1}$

$$
\delta\left(a_{i}\right)=\frac{a_{e i}-a_{i(i d)}}{a_{i(i d)}} ; \delta\left(d_{i}\right)=\frac{d_{e i}-d_{i(i d)}}{d_{i(i d)}}, i=0,1 .
$$

The symbolic form of coefficient errors (27), evaluated using MAPLE, contains positive and negative terms; i.e. there is possible to set them to zero. As proved in Ref. [7], three of the main errors can be zeroized simultaneously in combinations (28).

Note that the conditions (29) give less applicable results. The first set of conditions leads to the additional design equations for GIC elements in the form (30)

$$
\begin{aligned}
& R_{21}=\frac{Y_{11} R_{11} R_{31}}{Y_{11} R_{11}+2+Y_{21} R_{31}} ; R_{22}=R_{12} R_{31} \frac{Y_{22}+Y_{12}}{2} ; \\
& R_{32}=R_{21}+R_{22} .
\end{aligned}
$$

The computation was made under choice of $k_{1}=k_{2}=\omega_{0}$, in conformity with the recommendation published in Ref. [8]. It is important to point out the influence of the non-corrected $Q_{z}^{\prime}$ error, which causes magnitude frequency response distortion in the vicinity of frequency $\omega_{0}$, as will be shown in the numerical example. To avoid this, two ways can be used to the fully correct design:

a) A predistortion of $Q_{z}$ value, making final value errorless. This way is simple, because the error $\delta\left(a_{1}\right)$ is expressed by formula (31) evaluated under conditions (30). The improved form of design equations then includes the expressions (18) and (19) for transfer function coefficients together with optimization conditions (28). Note that the equation for coefficient $a_{1}$ in (18) is modified in the sense of the $Q_{z}$ predistortion to the form (32).

$\Delta\left(a_{1}\right)=a_{e 1}-a_{1(i d)}=\frac{\omega_{0}^{2} R_{31}}{B} ;$

$h \frac{\omega_{0}}{Q}=\frac{\alpha_{1}}{k_{2}} \frac{Y_{21}}{Y_{12}+Y_{22}}-\Delta\left(a_{1}\right) ; \quad \alpha_{1}=\frac{R_{21}}{R_{11}} ;$

$k_{2}=\frac{R_{12} R_{32} C_{42}}{R_{22}}$.

The circuit element design formulae computed by MAPLE give the resulting expressions (33). It is important to say that the "free" optional parameters $\left(R_{11}, R_{12}\right)$ influence circuit dynamic behaviour and $R_{31}$ affects frequency response. Unfortunately, the optimum values of these elements limit to zero and, from practical design point-of-view, their values should be chosen as small as possible.

b) The second way uses modified optimization conditions (28), or (29), which keep the $\omega_{0 p}^{\prime}$ and $\omega_{0 z}^{\prime}$ parameters errorless and make the $Q$-errors equal, i.e.

$$
\delta\left(d_{1}\right)=\delta\left(a_{1}\right) \Rightarrow \delta\left(Q_{p}^{\prime}\right)=\delta\left(Q_{z}^{\prime}\right)
$$

To avoid an additional group-delay error at frequency $\omega_{0}$ caused by $Q$-errors, the design can be combined with previously applied predistortion of coefficients $a_{e 1}$ and $d_{e 1}$.

The corresponding basic set of design equations is shown in (34).

$$
\begin{aligned}
& \delta\left(a_{0}=0 ; \delta\left(d_{0}\right)=0 ; \delta\left(d_{1}\right)=0 \Rightarrow \delta\left(\omega_{0 p}^{\prime}\right)=0 ; \delta\left(\omega_{0 z}^{\prime}\right)=0 ; \delta\left(Q_{p}^{\prime}\right)=0,\right. \\
& \delta\left(a_{0}=0 ; \delta\left(d_{0}\right)=0 ; \delta\left(a_{1}\right)=0 \Rightarrow \delta\left(\omega_{0 p}^{\prime}\right)=0 ; \delta\left(\omega_{0 z}^{\prime}\right)=0 ; \delta\left(Q_{z}^{\prime}\right)=0 .\right.
\end{aligned}
$$

\footnotetext{
1 As evident from (23), the coefficient errors indirectly express the errors of $\omega_{0}$ and $Q$ parameters as well.
} 


$$
\begin{aligned}
& Y_{10}=\frac{\omega_{0}^{2} Y_{22}(1-h)}{h} ; Y_{11}=\frac{\omega_{0} Y_{22}\left(B+B h+R_{31} Q \omega_{0}\right)}{h Q B} ; Y_{12}=\frac{Y_{22}(1-h)}{h} ; \\
& Y_{21}=\left(\frac{\omega_{0}^{3} R_{31} Q}{B^{2}} \frac{(2 h+1) \omega_{0}^{2}}{h}+\frac{(h+1) \omega_{0}}{R_{31} Q}\right) R_{11} Y_{22}+\frac{2 \omega_{0} Q}{B}+\frac{2 h}{R_{31}} ; \quad Y_{20}=\omega_{0}^{2} Y_{22} ; \\
& R_{21}=R_{11} R_{31} C_{41} ; R_{22}=\frac{R_{12} Y_{22} R_{31}}{2 h} ; R_{32}=R_{21}+R_{22} ; \\
& C_{41}=\frac{B \omega_{0} Y_{22}}{\left(R_{31} Q \omega_{0}^{2}+B(h+1) \omega_{0}\right) Y_{22} R_{11}+2 h Q B} ; \\
& C_{42}=\frac{\left(R_{31} Q \omega_{0}^{2}+B(h+1) \omega_{0}\right) Y_{22} R_{11}+2 h Q B}{\left(R_{31} Q \omega_{0}^{2}+B(h+1) \omega_{0}\right) R_{11} R_{12} Y_{22}+2 h B\left(Q R_{12}+\omega_{0} R_{11}\right)} \\
& k_{1}=k_{2}=\omega_{0} ; \quad Y_{22}, Y_{11}, R_{31}, R_{12} \text { - optional parameters. }
\end{aligned}
$$

$h \frac{\omega_{0}}{Q}=\frac{\alpha_{1}}{k_{2}} \frac{Y_{21}}{Y_{12}+Y_{22}}-\Delta\left(a_{1}\right)$

$\frac{\omega_{0}}{Q}=\frac{1}{k_{2}} \frac{Y_{11}-\alpha_{1} Y_{21}}{Y_{12}+Y_{22}}-\Delta\left(a_{1}\right)$

$h \omega_{0}^{2}=\frac{1}{k_{1} k_{2}} \frac{Y_{20}}{Y_{12}+Y_{22}}, \omega_{0}^{2}=\frac{1}{k_{1} k_{2}} \frac{Y_{10}+Y_{20}}{Y_{12}+Y_{22}}$,

$h=\frac{Y_{22}}{Y_{12}+Y_{22}}, k_{1}=\frac{R_{11} R_{31} C_{41}}{R_{21}}, k_{2}=\frac{R_{12} R_{32} C_{42}}{R_{22}}$,

$\alpha_{1}=\frac{R_{21}}{R_{11}}, k_{1}=k_{1}=\omega_{0}, \delta\left(a_{0}\right)=0, \delta\left(d_{0}\right)=0$,

$\delta\left(d_{1}\right)=\delta\left(a_{1}\right)$

A symbolic solution of the Eqs. (34) was made using MAPLE. The free design parameters are the same as in the case a), i. e. $Y_{22}$, $R_{11}, R_{31}$ and $\mathrm{R}_{12}$.

The discussed design procedure will be now demonstrated on the numerical example. Let us consider the frequency normalized allpass transfer function $H(s)$ assigned by parameters $h=1.0$, $\omega_{0}=1.0, Q=4.0$,

$$
H(s)=1.0 \frac{s^{2}-0.25 s+1}{s^{2}+0.25 s+1}
$$

proposed to the group-delay equalization in the denormalized frequency range $10^{\circ} \mathrm{MHz}$. The circuit realization presumes CFOA AD 844 as the active element. Normalized amplifier main parameters are $R_{T}=400, B=20$.

The simplest design version corresponding to the "basic" design equations (18), (19), (20) and (30) leads to the divider element values $Y_{10}=0, Y_{11}=0.50000, Y_{12}=0, Y_{20}=1.0, Y_{21}=8.50000$, $Y_{22}=1.0$; and GIC passive RC-network components $R_{11}=0.25$, $R_{21}=0.00735, R_{31}=0.25, C_{41}=0.11765, R_{12}=0.25, R_{22}=$ $=0.03125, R_{32}=0.03860, C_{42}=3.23809$.

An obtained circuit analysis confirmed correctness of design procedure and acceptable accuracy of the developed algorithm for transfer function order reduction. The evaluated parameters of the "full" and simplified transfer functions are summarized in the Table 1.

The non-zero parameter $\Delta\left(a_{1}\right)$ causes magnitude frequency response error $-0.446 \mathrm{~dB}$ at frequency $\omega_{0}=1$. Simultaneously group-delay error $\delta(\tau)=2.71 \%$ arises at the same frequency.

An improved design procedure using Eqs. (33) gives the following results under the same initial conditions (choice $R_{11}=R_{31}=$ $=R_{12}=0.25, Y_{22}=1.0$ ).

\begin{tabular}{|c|c|c|c|c|c|c|c|}
\hline Function & $\omega_{0 p}$ & $Q_{p}$ & $\omega_{0 z}$ & $Q_{z}$ & $Q_{z A B}^{\prime}$ & $\sigma_{p}$ & $\Delta\left(a_{1}\right)$ \\
\hline ideal & 1.0 & 4.0 & 1.0 & 4.0 & - & - & 0 \\
\hline "full" & 0.999982 & 4.000107 & 1.000001 & 4.210523 & - & - & 0.0124998 \\
\hline simplified & 0.999999 & 4.000000 & 1.000024 & 4.210475 & 4.210526 & 69.29936 & 0.012500 \\
\hline
\end{tabular}




\begin{tabular}{|c|c|c|c|c|c|c|c|}
\hline Function & $\omega_{0 p}$ & $Q_{p}$ & $\omega_{0 z}$ & $Q_{z}$ & $Q_{z A B}^{\prime}$ & $\sigma_{p}$ & $\tau(1)$ \\
\hline ideal & 1.0 & 4.0 & 1.0 & 4.0 & - & - & 16.00000 \\
\hline "full" & 0.999982 & 4.000107 & 1.000001 & 3.999997 & - & - & 16.01271 \\
\hline simplified & 0.999999 & 4.000000 & 1.000024 & 3.999951 & 4.000000 & 69.301956 & 16.01271 \\
\hline
\end{tabular}

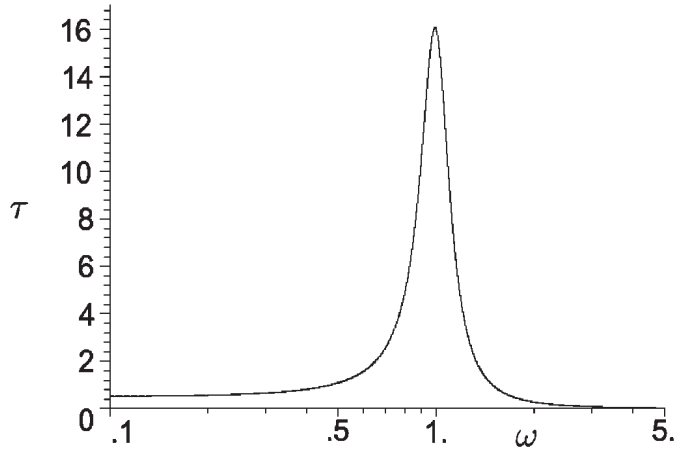

Fig. 6. Group-delay frequency response

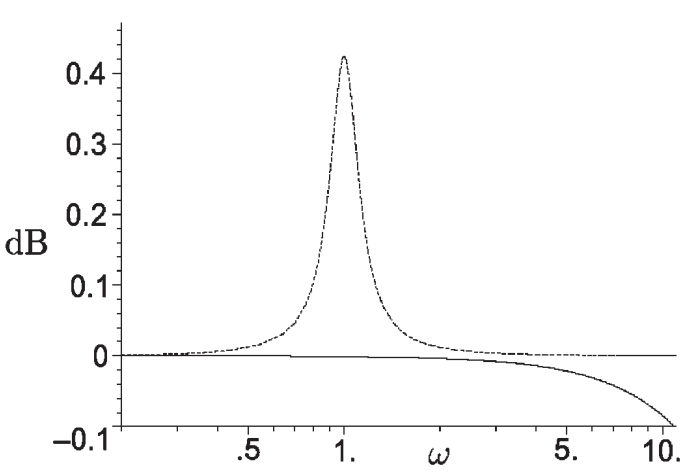

Fig. 7. Magnitude frequency response
Element values: $Y_{10}=0, Y_{11}=0.51250, Y_{12}=0, Y_{20}=1.0$, $Y_{21}=8.93813, Y_{22}=1.0 ; R_{11}=0.25000, R_{21}=0.00734, R_{31}=$ $=0.25000, C_{41}=0.11747, R_{12}=0.25000, R_{22}=0.03125, R_{32}=$ $=0.03859, C_{42}=3.23900$.

The corresponding magnitude a group-delay frequency responses are shown in Fig. 6, 7. In Fig. 7 the dotted line denotes the frequency response of the predistorted transfer function.

Class b) design, based on Eqs. (34) gives similar results. Divider admittance values are $Y_{10}=0, Y_{11}=0.52500, Y_{12}=0$, $Y_{20}=1.0, Y_{21}=4.26250, Y_{22}=1.0$, and GIC passive elements
$\mathrm{R}_{11}=0.25, R_{21}=0.015396, R_{31}=0.25, C_{41}=0.246334, R_{12}=$ $=0.25, R_{22}=0.0625, R_{32}=0.077896, C_{42}=3.20941$. Note that the design uses the same values of the optional elements to obtain comparable results to class a) versions. Similarly to the previous cases the designed circuit was simulated and analysis results are summarized in Table 3. For illustration, the $\tau$-error frequency response is shown in Fig. 8 and magnitude frequency response in Fig. 9. As it can be observed, the correction of magnitude frequency response is worse in comparison to the class a) design.

Parameters of the class b) design

Table 3

\begin{tabular}{|c|c|c|c|c|c|c|c|}
\hline Function & $\omega_{0 p}$ & $Q_{p}$ & $\omega_{0 z}$ & $Q_{z}$ & $Q_{z A B}^{\prime}$ & $\sigma_{p}$ & $\tau(1)$ \\
\hline ideal & 1.0 & 4.0 & 1.0 & 4.0 & - & - & 16.00000 \\
\hline "full" & 0.999614 & 4.000916 & 1.000002 & 3.999986 & - & - & 16.02677 \\
\hline simplified & 0.999637 & 4.000559 & 1.000049 & 3.999899 & 3.999999 & 34.608310 & 16.02677 \\
\hline
\end{tabular}

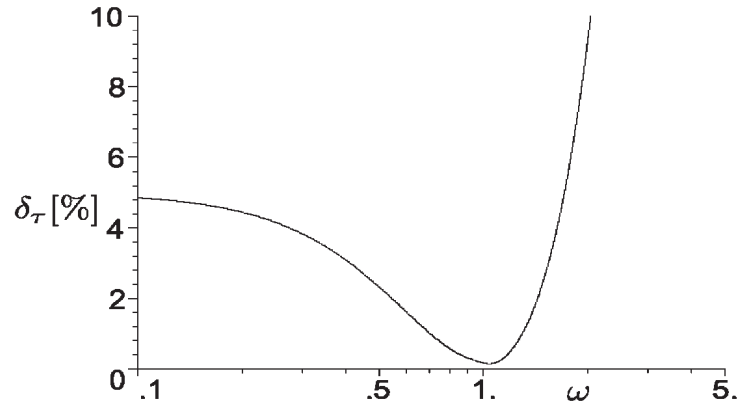

Fig. 8: Group-delay error response

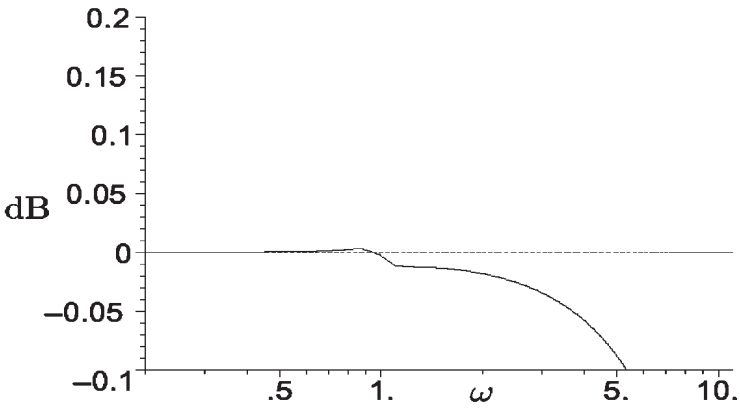

Fig. 9: Magnitude frequency response 


\begin{tabular}{|c|c|c|c|c|c|c|c|}
\hline Function & $\omega_{0 p}$ & $Q_{p}$ & $\omega_{0 z}$ & $Q_{z}$ & $Q_{z A B}^{\prime}$ & $\sigma_{p}$ & $\tau(1)$ \\
\hline ideal & 1.0 & 4.0 & 1.0 & 4.0 & - & - & 16.716 \\
\hline "full" & 0.998381 & 4.461324 & 1.000172 & 4.478866 & - & - & 17.912 \\
\hline simplified & 0.998212 & 4.457200 & 1.000232 & 4.479514 & 4.480210 & 14.426514 & 17.912 \\
\hline
\end{tabular}

Comparing the evaluated circuit parameters, it is possible to render some partial conclusions:

- Both the class a) versions give similar results.

- Sensitivity of transfer function parameters to the amplifier GBW is significantly influenced by suitable chioce of $R_{31}$ value. This fact is in agreement with general theory of current-feedback circuits. The lesser value of $R_{31}$ makes circuit frequency range wider.

- Class b) design leads to a higher sensitivity to the amplifier GBW, which is evident from the comparison of the first parasitic poles of the resulting "full" transfer function, or indirectly, by comparing $\sigma_{p}$ values. To improve the frequency properties and gain conformable results, it is necessary to reduce the $R_{31}$ value approximately by half.

The higher stage of the optimized design includes additional dynamic optimization in the sense of the equalization of amplifier maximum output voltages. Dynamic analysis disclosed inappropriate overshoot of the fourth amplifier output voltage in the vicinity of frequency $\omega=1$. To improve circuit dynamic properties, the set of design equations was extended about conditions (36) expressing the request of equal amplifier output voltages at frequency $\omega_{0}$

$$
\operatorname{Mod}_{A 1}\left(\omega_{0}\right)=\operatorname{Mod}_{A 2}\left(\omega_{0}\right)=\operatorname{Mod}_{A 3}\left(\omega_{0}\right)=\operatorname{Mod}_{A 4}\left(\omega_{0}\right) .
$$

Here $\operatorname{Mod}_{A i}\left(\omega_{0}\right),{ }_{i=1,2,3,4}$ expresses symbolically evaluated modul of partial transfer function

$$
\left|H_{A i}\left(j \omega_{0}\right)\right|=\left|\frac{V_{o i}\left(j \omega_{0}\right)}{V_{i n}\left(j \omega_{0}\right)}\right| \quad i=1,2,3,4
$$

corresponding to the amplifier outputs at frequency $\omega_{0}$.

A solution of the extended design equations provided the following results:

- The "full" set of equations containing Eqs. (33) and the additional dynamic conditions (36) is unsolvable, the requests to the $\omega_{0}$ - and $Q$-errors minimization negate dynamic equalization.

- The full dynamic optimization allows only $\omega_{0 z}$-error zeroing, the remaining errors are uncorrected. These can be minimized by a suitable choice of the optional value of $R_{32}$, limit value is $R_{32}$ $\rightarrow 0$

- Compromise solution including $\omega_{0 p}$ and $\omega_{0 z}$ errors zeroing and partial dynamic equalization seems to be the most acceptable. Optimum results were achieved considering conditions

$$
\operatorname{Mod}_{A 1}\left(\omega_{0}\right)=\operatorname{Mod}_{A 3}\left(\omega_{0}\right)=\operatorname{Mod}_{A 4}\left(\omega_{0}\right)
$$

Maximum output voltage of the $2^{\text {nd }}$ amplifier is in this case lower than in others.

- In general, dynamic optimization deteriorates frequency properties and leads to the higher errors of resulting group-delay and magnitude frequency responses. A fully acceptable solution is achievable using a more sophisticated optimization strategy, e.g. using evolutionary algorithms, or using a current-mode design.

A numerical illustration of the results obtained by using a compromise design is shown in table 4 . Here the optional parameters were chosen $R_{31}=0.125, R_{32}=0.75$ and $Y_{22}=1.0$. Note that the ratio $R_{32} / R_{31}$ influences circuit frequency properties and the chosen value corresponds to the local optimum. The calculated dynamic overshoots attain to $+13.5 \mathrm{~dB}$. An additional predistortion of transfer function improving the final parameters was not made, but it would be possible. Fig. 10 illustrates the results of the equalization of the amplifier maximum output voltages. As evident, the simplified approach gives acceptable accuracy of the dynamic optimization.

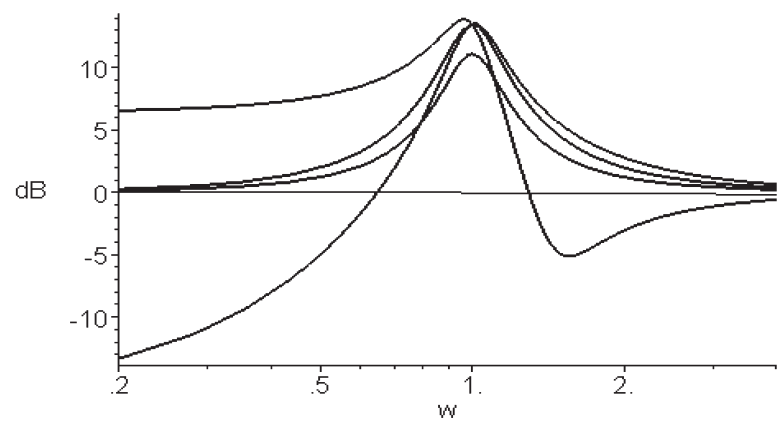

Fig. 10: Results of the dynamic optimization

\section{Conclusions}

The aim of this paper is to mention a new possibility of the allpass design. The described circuit is original, gained by the modification of the earlier published general divider structure. The use of CFOA warrants a wide frequency range, acceptable for design of phase equalizers in video- or fast-data-signal processing systems. The developed design procedures give improved solutions without noticeable group-delay and magnitude frequency response errors.

At this moment multicriterial optimization including circuit dynamics is not fully solved. This problem is a topic of the future research and its solution is posible using a current-mode design or by application of other variants of GIC circuits, e.g. GICs based on current conveyors. 


\section{KOMNIIKOCle}

\section{References}

[1] KVASIL, J.: Lineární obvody. NADAS, Praha, 1967.

[2] PLOSZEK, A., VOLNER, R.: Elektrické analogové obvody. VŠD Žilina, 1991, ISBN 80-7100-045-0

[3] HERPY, BERKA: Active RC Filters. Akademiai Kiado, Budapest, 1985

[4] SCHAUMANN, R., GHAUSI, M. S., LAKER, K. L.: Design of Analog Filters. Prentice Hall, 1990

[5] HASLER, M., NEIRINCK, J.: Electric Filters. Artech House, inc., Dedham, USA, 1986. ISBN 0-89006-186-6

[6] MARTINEK, P., TICHÁ, D., BOREŠ, P.: A Contribution to the Design of Filters Based on a Generalized Divider. In: Proc. of the ECCTD’97 Conference, Technical University Budapest, Budapest, 1997, Vol. 2, pp. 573-577 ISBN 963-420-523-2

[7] TICHÁ,D.: Integrované filtre v telekomunikačnej technike (in Slovak). PhD. Thesis, ŽU Žilina, 2000

[8] MARTINEK, P.: An Optimized Design of Circuits Containing GICs. Proc. of the 8th International Czech-Slovak Scientific Conference Radioelektronika 98, FEECS TU Brno,1998, Vol.1,pp. 42-45 ISBN 80-214-0983-5. 